\title{
Les éléments fondamentaux dans la poésie de \\ Philippe Jaccottet
}

Recherche proposée par

Ahmed Mohamed El-Halwagui

Maître de conférences à la faculté des Lettres- Université de Tanta

2019

\section{Introduction:}

Philippe Jaccottet est né en Suisse en 1925; ensuite il s'est installé en France définitivement depuis 1953. C'est un écrivain, critique littéraire et surtout poète et traducteur . 1944, date de la première publication de Jaccottet et en 2011, le poète publie sa dernière publication dans la collection Poésie: il s'agit de son anthologie, L'encre serait de l'ombre, qui regroupe des textes écrits entre 1946 et 2008.Parmi ses œuvres importantes: L'Effraie et autres poésies ( 1953), L'Ignorant ( 1958), Airs (1967), Paysages avec figures absentes (1976); Pensées sous les nuages (1983) et La Semaison (1984). Il était l'ami de Francis Ponge, André Du Bouchet, Yves Bonnefoy et Jacques Dupin. Bien plus, il traduit de l'allemand toutes les œuvres de Musil, Rilke, Hölderlin et bien d'autres. Il collabore à La Nouvelle Revue Française et publie presque toutes ses œuvres chez Gallimard. Pourquoi nous choisissons Jaccottet? Parce qu'il se classe parmi les grandes figures de la poésie française dans les trente dernières années. Il est couronné de plusieurs prix littéraires, comme poète, traducteur et critique littéraire.

Pour ne pas alourdir l'étude, nous visons d'étudier trois de ses œuvres importantes: Pensées sous les nuages, A La Lumière d'Hiver et Poésie préfacé de Jean Starobinski. Le choix des recueils étudiés n'est pas fortuit, il est motivé par plusieurs raisons: ces œuvres en fait représentent des étapes différentes dans l'entreprise poétique de Jaccottet, et les dates de parution de ces œuvres sont aussi différentes. Chez Jaccottet, Il y a un lien fort entre poésie, méditation et célébration du monde sensible. Chez lui, les minces événements de la nature se trouvent personnifiés et humanisés. Le poètepaysagiste, est hanté par les quatre éléments fondamentaux des philosophes de l'Antiquité, l'eau, l'air, le feu et la terre; mais Jaccottet les traite à sa propre manière.

En effet, la poésie moderne et l'imagination créatrice ont besoin d'un support matériel, celui que l'on trouve dans les quatre éléments fondamentaux de notre univers; même s'il s'agit de poétiser le monde sensible et abstrait, d'où le concept de l'imagination matérielle; qui sera la toile de fond de notre étude. Cette équation sera notre problématique de recherche: comment Philippe Jaccottet pourra réconcilier cette thématique ancienne (les quatre éléments) avec les courants modernes de la poésie dont il fait partie; pour qu'il confirme une place éminente dans la création poétique 
contemporaine; ou plus précisément, comment faire cette alliance entre un monde sensible et un autre concret : une telle question s'impose. L'étude suivra la démarche suivante : nous tenterons de traiter ces quatre éléments fondamentaux dans la poésie de Jaccottet selon la conception de l'imagination matérielle de Gaston Bachelard. Nous essayerons aussi de ranger et d'analyser les éléments selon leur rapport de dissemblance ou de ressemblance.

\section{L'air :}

Tandis que l'élément terrestre incarne une volonté d'enracinement et de concrétisation, la poétisation de l'air marque cette intuition d'échappement, d'ascendance, de purification et de transparence. De plus, la poésie de Jaccottet est en rapport perpétuel voire directe avec le monde et ses éléments. $\mathrm{Au}$ centre de ses vers, se trouvent donc le monde aérien et ses ressemblants; les éléments qui ayant trait à l'air sont multiples et primordiaux : la légèreté, la souplesse, la finesse, la transparence et la clarté. Dans le poème " Lune à l'aube d'été", cette idée s'exprime clairement:

"Dans l'air de plus en plus clair $\quad$ Scintille encore cette larme" 1

Ou dans " Au dernier quart de la nuit" où le poète fait de l'élément de l'air une phénoménologie:

"là où la terre s'achève $\quad$ Levée au plus près de l'air

(dans la lumière où le rêve Invisible de Dieu erre)"2

Réconciliation, passage d'un état concret (terre) à un autre abstrait (air lumière-rêve-invisible). Grâce à cette progression de l'image et à cette traversée du négatif, l'entreprise poétique de Jaccottet vise à réconcilier les dimensions opposées de l'univers comme par exemple le limité et l'illimité. Dans cette occurrence, l'élément abstrait, chez Jaccottet, n'a aucun sens et aucune importance sauf lorsqu'il s'associe avec un autre élément concret naturel. Cette hypothèse nous montre que Philippe Jaccottet propose dans sa poésie une voie médiane entre ces deux mondes. Uniquement, grâce à cette posture professionnelle, l'élément naturel-abstrait (l'air) devient perceptible. Cette idée s'affirme dans le poème (Paroles dans l'air), où le poète énonce:

"L'air si clair dit: " je fus un temps votre maison,

Puis viendront d'autres voyageurs à votre place,

Et vous qui aimiez tant ce séjour, où irez-

Vous? Je vois bien de la poussière sur la terre, ${ }^{\prime 3}$

Ce lyrisme tend à réorganiser le monde poétique à travers ses éléments fondamentaux. Il s'agit d'une multiplication de dépassement et de transfert: transfert d'un élément naturel - abstrait-fondamental (air-clair) à un autre élément concret, ayant les mêmes traits, (poussière sur la terre). De plus, dans une autre dimension et sur un autre niveau, ce transfert peut se faire de sa voix personnelle le (je) - " L'air si clair dit : "). La fusion totale du Moi personnel du poète avec la Nature et ses éléments fondamentaux: il s'agit de 
l'action de faire parler L'air. Enchevêtrement de sa voix, de ses paroles avec un élément fondamental ( $L$ 'air) ; afin d'arriver à une transparence totale et absolue. En effet, cette originalité représente la toile de fond sur laquelle se tient la voie poétique de Jaccottet. Il est tout à fait caractéristique et révélateur de mettre en exergue ce dépassement et cette progression vers l'annulation du Moi et l'union totale avec L'Air: "A présent, lampe soufflée, Main plus errante, qui tremble, Je recommence lentement dans l'air. ${ }^{.4}$

Tremblement, errance et incertitude afin d'arriver en fin de compte à cette transparence, par le moyen de s'identifier à L'Air. D'autre part, la transcription poétique de cette progression révèle un procédé fréquent chez notre poète: c'est l'identification à un élément fondamental ou plus généralement avec la Nature. Dans un autre endroit dans le même recueil, il dit:

" Encrassé par la suie du jour éteint. Je le franchis, c'est l'air limpide, taciturne,

J'avance enfin parmi les feuilles apaisées,

Je puis enfin faire ces quelques pas, léger

Comme l'ombre de l'air,,$"$ "

Dans cette dimension à la fois existentielle, philosophique et poétique; et dans cet univers transparent, allégé et métamorphosé, le poète métamorphose, non seulement sa voix et ses paroles, mais aussi lui-même: il devient définitivement un être aérien. À ce propos et tout au long de ses recueils, Jaccottet a recours de nouveau à l'image de l'alliance du Moi et de l'air, ou d'un autre élément fondamental. En effet, ceci constitue un leitmotiv dans l'œuvre de Jaccottet. Par ailleurs et dans cette perspective, il n'est pas anodin de constater que cette poétique de la légèreté, (quelques pas, léger, Comme l'ombre de l'air), représente un rêve de l'élévation, du dépassement, de l'insaisissable, de l'illimité, et de l'évaporation; comme l'affirme JeanPierre Richard: " heureuse, ou innocente, la poésie parvient à reproduire en elle un tel langage. A la fois légers et denses, j'allais dire denses de légèreté, les Airs de Jaccottet retrouvent quelquefois cette réussite d'un poids évaporé, d'une matérialité irradiante"

Le sommet de la création poétique, pour notre poète paysagiste, est d'exprimer une manière d'être au monde, de calquer le monde avec ses éléments; ayant la possibilité de pouvoir le réorganiser : cette reformulation du monde est la tâche de la poésie de tous les temps. Il sera utile, au cours de cette réflexion, d'évoquer un autre poème qui a pour titre (La promenade à la fin de l'été):

"En bas s'amasse l'épaisseur des morts anciens,

La précipitation de la poussière jadis claire.

La pétrification des papillons et des essaims,

En bas le cimetière de la graine et de la pierre,

Les assises de nos amours, de nos regards et de nos plaintes,"7 
Ce système d'opposition, chez Jaccottet, est généralement donné d'emblée. La lecture de ces vers est alors une ouverture à deux altérités : celle du monde minéral (poussière/pierre) et celle du monde aérien (papillons/essaims). Bref, les papillons symbolisent à la fois l'Air et la légèreté. Cette structure thématique transformante de Jaccottet tourne du sommet à la base et de la base au sommet: (La pétrification des papillons) explicite bien cette idée. La réflexion oscille donc entre le saisissable et l'insaisissable (des morts anciens/ le cimetière / nos amours). Le génie de Jaccottet est de pouvoir faire de sa poésie une poésie du dépassement voire une poésie de l'heureuse traversée du négatif. Puisque le poète pense que la poésie est une forme de résistance à l'absurdité de la vie. Une volonté d'un magicien de métamorphoser ce monde terrestre en un autre aérien à travers son rêve d'hauteur, d'élévation et d'ascension. Ceci pour arriver à une vie réelle, comme le déclare Gaston Bachelard:" la vie aérienne est la vie réelle; au contraire, la vie terrestre est une vie imaginaire, une vie fugitive et lointaine. Les bois et les rochers sont des objets indécis, fuyants et plats. La véritable partie de la vie est le ciel bleu, les "nourritures" du monde sont les souffles et parfums. ${ }^{18}$

A ce propos, il n'est pas anodin de remarquer que Jaccottet évoque dans sa poésie, une certaine prolifération et une certaine multiplication de l'élément fondamental (L'Air): à titre d'exemple, dans le poème (Paroles dans l'air), le poète construit une maison aérienne:

"L'Air si clair dit: " Je fus un temps votre maison,

Puis viendront d'autres voyageurs à votre place,

Et vous qui aimiez tant ce séjour, où irez-

Vous? Je vois bien de la poussière sur la terre,"9

De prime abord, on est frappé par le caractère apparemment dérisoire du thème de la suspension, lié fortement avec (L'Air); ce thème joue un rôle décisif dans la poétique de Jaccottet et aussi dans son labyrinthe cérébral.il s'agit d'une personnification de l'air, qui joue un double rôle: celui de la transparence (clair) et celui de la purification (poussière sur la terre). Un autre exemple dans le poème (Le Locataire), où nous avons une alliance expressive entre l'air, la maison, la suspension, la légèreté et la lumière:

"Nous habitons une maison légère haut dans les aires, (.....)

La lumières est bâtie sur un abîme, elle est tremblante, (.....)

Porte le locataire dans la terre, toi, servante!" 10

Il s'agit d'une double action d'enracinement (terre) et d'ascension (haut dans les airs).Certes, toute la poétique de Jaccottet se base sur non seulement ce mouvement d'ascension et de descente, mais aussi sur un mouvement contradictoire de verticalité et d'horizontalité qui, en fin de compte, perd son sens: c'est une forme d'écriture en spirale. Réciproquement, quelle que soit sa vitalité secrète, le thème de la suspension montre bien la volonté de 
Jaccottet de faire de ses vers une poésie qui oscille entre deux mondes opposés: le terrestre et le céleste. Dans (Lune à l'aube d'été), le poète ajoute:

"Dans l'air de plus en plus clair

Scintille encore cette larme

Ou fable flamme dans du verre

Quand du sommeil des montagnes

monte une vapeur dorée

Ces merveilleux vers ci-dessus montrent bien l'importance du thème de la suspension dans la poétique de Jaccottet; ceci montre bien l'envie et la volonté de mettre les choses hors du temps et l'espace. La même idée des (montagnes), qui représentent la hauteur, la supériorité, l'accès vers la lumière et l'air pur à l'opposé de l'infériorité et l'obscurité du monde terrestre. Ici, il s'agit d'un double poids de L'Air : un poids léger (air/clair) et un poids lourd (vapeur dorée); et aussi un double mouvement du même élément fondamental: un mouvement du bas des (montagnes) vers le haut (monte) et vice-versa. D'ailleurs, L'Air peut aussi s'associer à la Lumière:

"Des lumières dans l'air et d'autres dans les glaces,

Des gens qui passent et d'autres immobiles, (...) Pour tisser un voile de $v^{\prime \prime} e^{12}$

Réflexion logique des lumières à travers les glaces. Chez Jaccottet, L'Air doux égale la lumière et la limpidité; par contre, nous avons une autre image de l'Air violent et agité, c'est le moment où (l'air) devient (vent) ou (souffle):

"Toute à la fin de la nuit Quand ce souffle s'est élevé (...)

Le vent le sait, qui traverse les fleuves"13

Lorsque la situation s'aggrave, la progression de l'image de l'air se dessine logiquement: à cette image du (souffle) qui devient (vent); répond une autre image métamorphosée de la brume, de l'ombre et de l'obscurité:

" et l'autre rive prise dans la brume Ou elle-même brume, ou pire: abîmeDans ce vent barbelé, (.....) Le pire froid, la pire ombre ne sont bientôt plus (.....)Par les sombres parois grandit et gagne,"14

Il s'agit d'une progression de l'image et d'une alchimie de l'air: (air-ventbrume).Le poète renforce l'image de l'obscurité (brume): il s'obstine à nous donner à voir un tableau où règne la moindre clarté (sombres).il est temps que Jaccottet compose des vers qui reflètent un état d'illusion, d'hallucination, mais aussi de soupçon; ainsi déclare Jacqueline Michel: " Jaccottet dans son application à écrire l'intervalle, ne "jardine-t-il pas nos ombres"? ne "rognet-il pas l'horizon" où s'accumule la brume, lorsqu'il demande à ses mots d'exprimer le soupçon doublé de silence,"15

\section{La terre :}

$\mathrm{Au}$ fur et à mesure, nous voulons montrer que le discours poétique de Jaccottet est basé, dans un premier temps, sur un système d'opposition; et dans un deuxième temps, sur le but d'associer et d'unir toutes les 
contradictions de la vie avec ses éléments. Cette poétique représente, en quelque sorte, la cellule matricielle, non seulement de la poésie de Jaccottet, mais aussi de la poésie contemporaine en générale. Apparemment, l'élément de la terre est à l'antipode de l'air: dans l'imagination des poètes, la terre c'est l'encrage voire l'enracinement dans le terrestre, tandis que l'air symbolise toutes les dérivations du rêve et de l'imaginaire: rêve de la hauteur, de l'élévation; mais, ça peut aussi être un rêve de la chute:" les métaphores de la hauteur, de l'élévation, de la profondeur, de l'abaissement, de la chute sont par excellence des métaphores axiomatiques. (.....)la chute profonde, la chute dans les gouffres noirs, la chute dans l'abîme, sont presque fatalement les chutes imaginaires en rapport avec une imagination (...) de la terre ténébreuse. (L'imagination de la chute comme une sorte de maladie de l'imagination de la montée, comme la nostalgie inexpiable de la hauteur."16 Dans cette occurrence, le rêve de la chute, chez Jaccottet, incarne, non seulement, un retour inévitable vers l'élément minéral qui est la terre, mais aussi son obstination de s'adhérer au monde réel. Bien que, Les quatre éléments fondamentaux sont formellement hétérogènes, or dans l'imagination de Jaccottet les choses cessent, par intervalles, de s'opposer:

"On ne vit pas longtemps comme les oiseaux Dans l'évidence du ciel, Et retombé à terre, On ne voit plus en eux précisément que des images

Ou des rêves. ${ }^{17}$ La vie aérienne rêvée est courte (on ne vit pas longtemps); même si elle représente la vérité purifiée (évidence). Mais également, cette direction vers le bas (la chute) est fatalement conçue (retombé à terre).D'ailleurs, la surimpression du thème de la chute du ciel vers la terre, est peut-être liée avec une conception religieuse: la chute du Paradis vers la terre: châtiment du (pêcher initial). En somme, nous pouvons dire que toute la poésie de Jaccottet est centralisée sur l'affrontement en quelque sorte de deux structures thématiques: aérien supérieur et terrestre inférieur. Bref, une poésie de la condition humaine et l'hypothèse du souhait. Une deuxième possibilité qui énonce une certitude, c'est que ces rêves d'élévation et de chute représentent un complexe et un syndrome, dans l'entreprise poétique de Jaccottet. Il sera utile, au cours de la réflexion, d'évoquer d'autres caractéristiques de la terre:" la terre est de nature essentiellement contradictoire, le lieu où la vie et la mort se croisent, et l'herbe apparaît en définitive comme la ressuscitée. ${ }^{18}$ Pareillement à l'élément de l'air, la terre elle aussi a un visage changeant dans l'imaginaire de Jaccottet. Ainsi, nous pouvons toucher et sentir cette poésie de l'immédiateté de la rêverie et de la contrariété. Il me semble tout à fait important de citer, à titre d'exemple, quelques aspects changeants de la terre dans la poésie de Jaccottet:

"Repos d'une heure sur les basses tables de la terre.

Paroles sans beaucoup d'écho. Lueurs de lierre.

Nous marchons entourés des derniers oiseaux de l'automne"19 
Dans cette atmosphère de sérénité et de repos, Jaccottet fait l'alliance entre la terre et la tranquillité. Dans un autre endroit, il rajoute: "Mais peut-être, plus légère, (...) Est-elle celle qui chante Avec la voix la plus pure Les distances de la terre" ${ }^{120}$

Ici, il s'agit d'une terre apaisante; elle peut à son tour transmettre une certaine légèreté voire une certaine pureté. Par conséquent, c'est une image de la terre qui chante en offrant du bonheur au poète. Par ailleurs, le choix des éléments naturels, spatio-temporels couvrent les poèmes de Jaccottet. Voici une autre image d'une terre troublée mais purifiée à l'aide d'autres éléments naturels:

"Ombres calmes, buissons tremblant à peine, et les couleurs, Elles aussi, ferment les yeux. L'obscurité $\quad$ Lave la terre." 21

A ce stade, les éléments fondamentaux vont exercer une influence réciproque (apaisante ou troublante) les uns sur les autres. Par contre, voici une autre image différente de l'élément minéral:

"Sans secours où qu'il se tourne, $\quad$ Acculé, cloué, vidé. Il ne pèse presque plus La terre qui nous portait tremble." 22

Terre tremblante qui peut transmettre (portait) une vibration tout au fond à l'intérieur de notre poète. C'est l'une des contradictions phénoménologiques les plus manifestes dans la poésie de Jaccottet. Corrélativement, cet élément minéral porte en lui la vie et la mort en même temps. Il peut être un paradis ou un enfer. Jaccottet a réussi à nous donner à voir cette polysémie de la terre :

"Auprès du mort, une barque de terre: (...)

Longuement autrefois j'ai regardé ces barques des tombeaux (...)

Ni d'aucun baume, ni d'aucune carte des Enfers. "23

En définitive, cette terre joyeuse peut se transformer en un objet de la mort:

"Au lieu où ce beau corps descend dans la terre inconnue,

Combattant ceint de cuir ou amoureuse morte nue,(...)

Nul ne peut séparer feu et cendre, rire et poussière, (...)

Ainsi l'homme nourri de la terre funèbre."

On veut montrer que le discours poétique de Jaccottet est centralisé sur cette nuance et cette multiplication de la conception donnée aux éléments fondamentaux. Terre paradis ou terre (inconnue et funèbre), ou bien terre prospère ou terre déserte:

"Des plumes blanches de vos ventres,

Longs miroirs des routes désertes, des fossés,

Terre de plus en plus visible et grande, tombe Et déjà berceau des herbes." 25

Dans les deux exemples précédents, notre poète vise à élargir le champ lexical de la terre: (poussière, cendre, routes, fossés, berceau, etc.) chacune de ces connotations peut nous donner à voir une image de la terre rêvée et 
conçue par le poète. Encore une fois, nous sommes devant une architecture poétique sonore et visuelle à la fois:

"Et par une autre oreille que la terre grande ouverte

Soit recueilli, plus haut, non pas plus haut,

Ailleurs, pas même ailleurs: soit recueilli"26

Il s'agit d'une poésie humaniste qui présente des sensations de nature différente : vue, ouïe, toucher, odorat: toutes sont convoquées simultanément. La terre peut elle aussi s'associer aux effets des sensations auditives et visuelles:

"Où est l'œil de la terre Dispersées, on voit mieux l'étendue

Nul ne le sait(...)
De l'avenir

L'oil de la terre est clairvoyant, par lequel le poète aperçoit et guette son avenir. À ce propos, il y a sans doute chez Jaccottet une sorte d'enchevêtrement entre différents thèmes poétiques. Chez lui, les sensations sont simplement juxtaposées et évoquées. Nous ne pouvons pas ne pas constater que l'expérience poétique de Jaccottet évoque irrésistiblement les quatre éléments fondamentaux séparés ou plutôt unifiés:

"La terre tout entière visible Mesurable Pleine de temps Suspendue à une plume qui monte De plus en plus lumineuse" ${ }^{28}$

Lorsque la terre s'unifie à l'air, nous avons une image de la (terre suspendue et lumineuse). Et dans cette perspective, l'alliance entre la terre et l'eau peut offrir une image de la terre humide, capable de donner naissance à l'élément végétal si important et inévitable dans l'imaginaire de Jaccottet:

"La terre ici montre la corde. Mais qu'il pleuve

Un seul jour, on devine à son humidité ${ }^{129}$

Ou dans un autre recueille: $\quad$ "Peut-être plus bas, comme une eau Qui s'enfonce dans la poussière du jardin.

Comme le sang qui se disperse, fourvoyé, dans l'inconnu. ${ }^{130}$

Désormais, la terre survivra par l'eau; et le jardin à son tour fleurira et prospérera. L'eau est nécessaire pour la terre comme le sang pour l'homme. A l'orée de ces différentes hypothèses réflexives, nous pouvons dire que la terre est de nature essentiellement contradictoire; et sa contradiction représente une source profonde d'inspiration pour notre poète.

\section{L'eau :}

Essentiellement, l'eau est l'élément fondamental de la vie. De plus, elle représente une source d'inspiration par excellence pour les poètes dans tous les temps. Pour Jaccottet, Il est bel et bien impossible de dissocier en lui le sentiment et la sensation de la transparence et de la pureté reflétées par l'eau. Bien plus, dans la rêverie poétique de Jaccottet, l'élément de l'eau est irremplaçable; cette rêverie a un rapport simple avec le réel; cette immédiateté et cette précarité représentent une source de joie, de poésie, et 
tout simplement de vie. Tout d'abord, le poète nous offre une image miroitante de l'eau:

"Pendant que je t'écoute, Le reflet d'une bougie

Tremble dans le miroir Comme une flamme tressée à de l'eau. ${ }^{\prime \prime 1}$

Le miroir est un outil de la rêverie poétique. Ici, il reflète l'image du poète à travers le mouvement qui berce l'eau. Lorsque le (je) s'unifie à l'eau, cette opération peut participer à dessiner un tableau de la vie onirique qui règne dans le poème; ou bien, dans une analyse psychanalytique, Bachelard ajoute: "Mais Narcisse à la fontaine n'est pas seulement livré à la contemplation de soi-même. Sa propre image est le centre d'un monde. Avec Narcisse, pour Narcisse, c'est toute la forêt qui se mire, tout le ciel qui vient prendre conscience de sa grandiose image." ${ }^{32}$ Ainsi, la volonté de regarder l'eau-miroir, c'est un désir et un plaisir à la fois. En effet, ce sont trois éléments qui permettent cette centralisation du moi: le poète, le miroir et bien évidemment l'eau :"s'il existe; le "je" de la narcose- s'il garde valeur d'individualité; le "je" de la rêverie, maintenu dans une telle vigilance qu'il peut se donner le bonheur d'écrire. ${ }^{133}$ Certainement, cette individualité et cet égocentrisme poussent le poète à voir son double reflété par l'eau; et à ce moment, il peut s'exprimer heureusement. Philippe Jaccottet nous propose comme d'habitude, une multiplication de l'imagination matérielle de l'eau, selon la conception de Bachelard. D'ailleurs, Jaccottet évoque l'eau comme un élément féminin par excellence:

" Regarde-la courir sur ses jambes toutes nouvelles

A la rencontre de l'amour Comme un ruisseau de verre tintant sur les roches,

Est-ce le fouet des hirondelles sur les prés humides Qui la presse?"34

Il s'agit ici d'un enchevêtrement entre plusieurs thèmes poétiques: la femme, la nudité et l'eau; c'est simplement l'image sensorielle de l'eau-sexe. Ces vers s'inscrivent dans une tradition poétique à la fois littéraire et picturale où règne un décor complètement naturel. Sur ce point de vue, Bachelard pose une question: "Quelle est donc la fonction sexuelle de la rivière? C'est d'évoquer la nudité féminine.(...) par conséquent, la femme qui s'y baignerait sera blanche et jeune; par conséquent elle sera nue. L'eau évoque d'ailleurs la nudité naturelle, la nudité qui peut garder une innocence. ${ }^{135}$ Ainsi, le rôle sexuel joué par l'eau a une double fonction: d'abord, la femme qui fascine, offusque et séduit le poète; deuxièmement, l'eau symbolise la mère qui berce tout en donnant une nouvelle naissance à l'homme en général, et au poète contemplant en particulier:

"Même au bord des rivières transparentes et même à l'aube, Mais en me forçant à parler, plus têtu

Que l'enfant quand il grave avec peine son nom sur la table d'école, " 36 
L'eau devient transparente lorsqu'elle s'associe à la femme et à la mère. Dans cette union, le poète se trouve forcé de convoquer ses souvenirs d'enfance. Sur le rôle de l'eau-mère, Bachelard affirme: "des quatre éléments, il n'y a que l'eau qui puisse bercer. C'est elle l'élément berçant. C'est un trait de plus de son caractère féminin: elle berce comme une mère (...) l'eau nous porte. L'eau nous berce. L'eau nous endort. L'eau nous rend notre mère. ${ }^{137}$ Globalement, nous pouvons dire clairement que l'essentiel de la symbolique de l'eau, c'est qu'elle est une source à la fois imaginaire et réelle de vie. A son tour, cet élément initial peut jouer de multiples rôles extraordinaires: c'est un moyen de purification, un lieu de régénérescence, un facteur de survivance, de transparence et d'innocence.

"En ce jardin la voix des eaux ne tarit pas,

Est-ce une blanchisseuse ou les nymphes d'en bas,

Ma voix n'arrive pas à se mêler à celles (...)

-Les nymphes, les ruisseaux, images où se complaire! (...)

Une fille cachée? Je n'ai rien inventé: ${ }^{\prime 138}$

Remarquons qu'il y a une fusion totale entre la voix du poète et la voix des eaux. Mais, le poète est incapable de faire ce mélange entre lui et cette femme idéalisée voire imaginaire (les nymphes/ cachée). Car, selon Jaccottet, cette union ne peut se faire qu'avec uniquement un élément imaginaire (les nymphes) et un autre élément naturel (les ruisseaux); d'où vient le concept de l'imagination matérielle dans l'analyse de la poésie. "Ce qui fascine Jaccottet ...dans les figures des nymphes, c'est qu'elles nous ouvrent au sacré, d'où sa nostalgie pour " cette beauté qui est équilibre entre le sol et le ciel, la nuit et le jour, ce moment où une femme, suspendue entre deux absences, entre deux domaines d'ombre, est éclairée par l'Illimitéé ${ }^{\prime 39}$ Un parcours de la poésie de Jaccottet nous permettra de mettre en évidence cette fécondité donnée à l'image de l'eau. D'ailleurs, le poète suisse s'efforce de faire de sa poésie une poésie de l'intervalle: son idéal poétique se trouve à cheval entre deux mondes bien séparés, différents et même contradictoires. Bref, on trouve chez lui, que le sacré peut être facilement mêlé au quotidien, et cette opération est due peut-être à l'influence du surréalisme, qui est une de ses nombreuses références.

"Des femmes crient dans la poussière. Car chanter, (...)

Mais toujours, sur ces gouffres d'eau, luit l'éphémère. (...)

Pays poursuivi par l'eau, Comme la femme nocturne,

D'une manière générale, nous assistons à une progression en ce qui concerne l'image de l'eau: dans un premier temps, l'eau fascine (femme); mais dans un deuxième temps, elle peut aussi détruire (gouffres d'eau). A l'orée de cette connotation érotique donnée à l'image de l'eau, on peut dire que cet élément fondamental ne cesse de se développer:

"Toute à la fin de la nuit quand ce souffle s'est élevé 
Une bougie d'abord a défailli Le vent le sait, qui traverse les
fleuves

Ici, il s'agit d'une eau inquiétante et même mortelle. Par intervalles, cet élément se débarrasse de son rôle ancien: purificateur, apaisant et régénérateur. En effet, le symbolisme poétique de Jaccottet atteint son apogée; l'eau, au lieu de donner vie et naissance nouvelles, elle est, par contre, mourante et mortelle; tout en traversant les (fleuves) dans une nuit nocturne (bougie), pour arriver à la rive de la mort. C'est l'une des figurations et des convictions de la mythologie religieuse de l'ancienne Égypte:

" Auprès du mort, une barque de terre: Le Nil va-t-il couler jusqu'à ce cour?

Longuement autrefois j'ai regardé ces barques des tombeaux (...)

Ni d'aucun baume, ni d'aucune carte des Enfers. ${ }^{142}$

Ainsi s'explique une irrésistible obstination de la part de Jaccottet; lorsqu'il s'agit d'une évocation du gouffre de la mort, il convoque aussi et en même temps l'image des barques qui doivent traverser la rivière de la mort; pour arriver en fin de compte à l'autre vie. De plus, il s'agit d'un passage ou plutôt un voyage vers l'inconnu, ayant la possibilité d'une communication directe avec les morts; sans avoir une connaissance exacte de ce voyage fluvial (ni d'aucune carte des Enfers): " voici donc pourquoi l'eau est la matière de la mort belle et fidèle. L'eau seule peut dormir, en gardant la beauté; l'eau seule peut mourir, immobile, en gardant ses reflets. (....) l'eau qui est la patrie des nymphes vivantes est aussi la patrie des nymphes mortes. Elle est la vraie matière de la mort bien féminine." ${ }^{43} \quad$ En définitive, cette contexture entre un élément féminin (nymphes), un élément naturel (rivière), un élément fondamental ( $\left.l^{\prime} e a u\right)$ et un élément à la fois réel et imaginaire (la mort), peut nous donner une métaphore nostalgique de l'imagination matérielle de Philippe Jaccottet et aussi de son idéal poétique. Il s'agit donc d'une poétique de la rêverie, du dépassement et du surnaturel:

"Même invisible, je t'ai reconnu, Tisserand des ruisseaux surnaturels. ${ }^{\text {"44 }}$

Donc, tout est permis, puisque l'auteur assimile l'eau au surnaturel. À la genèse, l'eau est toujours purificatrice, mais par intervalles dans l'imagination poétique, elle peut jouer un rôle antithétique. Elle est plutôt inquiétante et troublante:

"Le fleuve craquelé se trouble. Les eaux montent

Et lavent les pavés des berges. Car le vent

Comme une barque sombre et haute est descendu

De l'Océan, chargé d'un fret de graines jaunes.

Il flotte une odeur d'eau, lointaine et fade... On tremble, 45

L'eau troublée sur laquelle flotte une barque sombre: cette image triste et désespérée reflète l'état d'âme et l'état d'esprit du poète tremblant qui 
guette cette scène sur la rive. "L'eau est alors un néant substantiel. On ne peut aller plus loin dans le désespoir. Pour certaines âmes, l'eau est la matière du désespoir." ${ }^{46}$ L'eau triste, l'eau morne, l'eau courante et l'eau fermée; ceci nous pousse en fait d'analyser une dernière image de l'eau: à savoir l'eau douce fluviale et l'eau des mers.

"La mer est de nouveau obscure. Tu comprends, (...)

Où s'écroulent les rocs, la mer est noire, et tonne

Dans sa cloche de pluie. Une chauve-souris" 47

Les fleuves, les rivières et les ruisseaux avec leur eau douce sont une préférence dans la poésie de Jaccottet. Chez lui, l'eau fluviale est la plupart du temps tranquillisante. Par contre, la mer est quasi absente de ses œuvres. Poétiquement, l'eau douce a la supériorité: " La suprématie de l'eau terrestre sur l'eau marine n'a naturellement pas échappé aux mythologues modernes. ${ }^{118}$ Certainement, les mythologues modernes sont bien évidemment les poètes contemporains comme Jaccottet; puisqu'ils traitent dans leurs poésies des thèmes que l'on rencontre dans les traditions philosophiques les plus anciennes; comme les quatre éléments fondamentaux. Mais, scientifiquement, du point de vue du même critique, l'eau marine est l'origine; elle est eau-de-vie par excellence: "la science nous dit que la vie réelle a commencé dans la mer; c'est dans une sorte d'océan céleste que commence la vie rêvante." ${ }^{\prime 49}$

\section{Le feu:}

En effet, l'originalité poétique de Philippe Jaccottet se caractérise dès les débuts par sa vocation pour un monde bucolique, plein d'éléments contradictoires. Dans cette optique, nous préférons analyser l'élément du $(f e u)$, juste après avoir traité celui de (l'eau); qui sont antithétiques voire ennemis; selon le rationalisme psychanalytique de Jaccottet et de Bachelard: "En particulier, l'eau et le feu restent ennemis jusque dans la rêverie et celui qui écoute le ruisseau ne peut guère comprendre celui qui entend chanter les flammes: ils ne parlent pas la même langue. ${ }^{\prime 50}$ Mais, quand même, tout est possible par intervalles dans un monde poétique, le rôle de la poésie, même s'il tente de calquer la réalité, il la décrit à travers une image enchantée, onirique et parfois hallucinée. Et c'est là exactement le génie de l'acte poétique. A cet égard, nous trouvons que la poésie moderniste de Jaccottet peut prendre, de temps en temps, les légendes comme modèles et comme des sources d'inspiration et de méditation:

" (et les papillons sont autant de flammes perdues, (...)

Les cris d'oiseaux lointains en sont les nouds, (...)

Elle m'enflamme. (...) (...) et nous laissent moins de cendres

Que feu d'un soir au foyer, (...) tel le soleil dans notre dos encore ${ }^{\prime \prime 51}$

Ainsi, la thématique du feu est liée tout simplement au complexe du Phénix. Il s'agit d'un oiseau légendaire géant, qui prend sa source de la 
mythologie persane. A la fin de sa vie, il s'enflamme et se transforme en cendres; de cette cendre, cet oiseau peut renaître à nouveau. C'est une symbolique très forte de la résurrection; par l'aide du soleil qu'il adore. "Le Phénix des poètes explore en paroles enflammées; enflammantes. Il est au centre d'un champ illimité de métaphores. Une telle image ne peut pas laisser l'imagination tranquille. (....) ainsi l'écrivain a choisi l'oiseau de feu pour mettre le point final à son enthousiasme littéraire. ${ }^{\prime 52} \quad$ A la manière de cet oiseau de feu, notre poète veut nous démontrer, dans un moment désespéré, à quel point cet élément fondamental (le $f e u$ ) est capable de nous donner une autre nouvelle vie. Dans l'imagination matérielle, au cas où le poète décide de mettre fin à sa vie ou à son œuvre poétique; celui-ci se trouve obligé d'avoir recours au feu; pour sa force rénovatrice: "leur corps est cendre,

Cendre leur ombre et leur souvenir; la cendre même,

Un vent sans nom et sans visage la disperse

Et ce vent même, quoi l'efface? (...) Ces cris d'oiseaux sous les nuages ${ }^{\prime \prime 53}$ Ainsi, le corps devient cendre par le feu; et cette cendre est dispersée par le vent. En effet, ces étapes progressives nous donne à voir l'image de l'incarnation du Phénix enflammé qui souffre: (cris d'oiseaux). A cette occurrence, il nous semble que Jaccottet croit momentanément au bouddhisme et à l'hindouisme; en ce qui concerne la réincarnation des esprits. En outre, une étude plus poussée dans cette voie nous permettrait de suivre la poursuite de notre poète à d'autres légendes:

" Comme l'huile qui dort dans la lampe et bientôt; (...)

Tu murmures et tu brûles. (Mais comment dire

Cette chose qui est trop pure pour la voix?)

Tu es le feu naissant sur les froides rivières, ${ }^{154}$ Ou autrement dit:

" Ô compagne du ténébreux entends ce qu'écoute sa cendre afin de mieux céder au feu (...) peut-être la clarté divine ${ }^{155}$

Après avoir filé la métaphore de l'oiseau-feu (Le Phénix), le poète remonte un degré pour nous offrir l'image du poète-feu: c'est le complexe d'Empédocle. Naguère, c'était l'oiseau qui se sacrifiait; mais désormais, c'est le poète lui-même qui se donne au feu pour deux objectifs: le premier est d'avoir une purification (trop pure) ou (clarté divine); le deuxième s'agit en quelque sorte d'un anéantissement facultatif pour se donner à lui-même et au monde entier une nouvelle naissance. "Cette rêverie très spéciale et pourtant très générale détermine un véritable complexe où s'unissent l'amour et le respect du feu, l'instinct de vivre et l'instinct de mourir. Pour être rapide, on pourrait l'appeler le complexe d'Empédocle." ${ }^{56} \quad$ Empédocle était un philosophe et un poète grec; il voulait prouver à ses sympathisants, en se jetant dans le volcan d'Etna, que le feu est purificateur et rédempteur. Désormais, cette action sera pour toujours une symbolique de l'anéantissement, du sacrifice et bien sûre de la poétique du feu. 
Jaccottet, en nous présentant le symbole d'Empédocle, veut à son tour reformuler l'image du poète et ses relations avec le monde qui l'entoure. " Empédocle est une des plus grandes images de la poétique de l'anéantissement. Dans l'acte empédocléen l'homme est aussi grand que le feu. L'homme est l'acteur d'un cosmodrame vrai. (...) le sacrifice d'Empédocle vient aider à la purification du monde, à la divinisation de l'élément pré-divin." ${ }^{157} \quad$ Donc, la poétique du feu est une poétique humanitaire par excellence; puisque le grand acteur de cette poétique est l'homme et surtout l'homme-poète. Et cette fusion totale entre le poète le feu peut donner une vie nouvelle.

"Moi, poète abrité, $\quad$ épargne, souffrant à peine, (...)

A présent, lampe soufflée, main plus errante, qui tremble, Je recommence lentement dans l'air." ${ }^{58}$

Une combinaison entre le poète et le feu peut refléter les caractéristiques de l'un sur l'autre. Par conséquent, le feu devient personnifié et humanisé, et le poète, à son tour, peut, par la puissance du feu, recommencer de nouveau ou à nouveau. A force de la présence du feu et de ses synonymes, on peut dire que la poésie de Jaccottet est une poésie enflammée et scintillante. Ce qui importe précisément, c'est que la question des complexes du feu soit posée encore une fois. "Les montagnes autant de fumées (...)

Je ne vois presque plus rien que la lumière (...)

Toute la montagne du jour est allumée,

elle ne me surplombe plus, Elle m'enflamme. ${ }^{.59} \quad$ Ou dans un autre endroit:

"sera bientôt vêtu de feux dans les ténèbres

Ou de fleurs dans le jour une nouvelle fois?

Ainsi l'homme nourri de la terre funèbre."

Il y a toujours une lutte entre la lumière du feu et l'obscurité de la vie. Chez Jaccottet, ce feu-lumière fondamental est capable de donner espoir pour l'homme; contre les ténèbres des éléments naturels. Par ailleurs, on peut rajouter un denier complexe concernant le feu: à part les complexes du Phénix et celui d'Empédocle, nous avons le complexe de Prométhée: le voleur du feu ou l'éclair de la montagne des dieux, qui voulait, selon la légende grecque, donner à l'homme les traits du feu: la chaleur, l'enthousiasme et la suprématie. Le nœud de Prométhée résume clairement le sens de la poésie humanitaire de Jaccottet: "nous allons considérer une image de base du prométhéisme: Prométhée, héros qui a volé le feu du ciel pour le donner aux hommes (...) le Prométhée poétique nous invite à une esthétique de l'humain. ${ }^{\prime \prime 6 l}$ En effet, ces complexes du feu seront désormais un vrai syndrome qui caractérise la poétique de Jaccottet. Dorénavant, cet élément fondamental (le feu) sera un leitmotiv dans l'imagination poétique de ce poète-humaniste. Dans une autre optique, l'imagerie du feu, chez Jaccottet, 
prend trois dimensions: l'anéantissement, l'effacement et la purification (Empédocle), c'est un défi contre la nature et ses éléments; puis le sacrifice et le dépassement (Le Phénix); ensuite, l'amour pour l'humanité (Prométhée), c'est un défi contre les dieux. Au surplus, on peut ajouter à cette imagerie le feu-désir ou plutôt le feu-sexe.

" Car le feu a encore une splendeur, même s'il ruine,

Il est rouge, il se laisse comparer au tigre

Ou à la rose, à la rigueur on peut prétendre,

On peut s'imaginer qu'on le désire comme une langue ou comme un corps:

Et d'une flamme soudain plus haute et plus vive

Illuminer la chambre jusqu'au lit ou au jardin" 62

Cet exemple peut nous donner à voir deux autres images du feu: le feusublimation-majesté (splendeur), et le feu comme un langage du corps féminin (langue-corps). Devant cette scène poétique, Jaccottet devient, bon gré mal gré, un homme à genoux: une soumission, une remise et une obéissance totales vis-à-vis du feu et de la femme. Cette ambivalence du feufemme offre à l'imagination une couleur vivante (rouge); elle est en somme un désir personnifié (tigre). C'est le désir ardent qui peut illuminer la chambre et le lit.

" des beaux pieds nus- découvre la femme d'ébène

Et de cristal, la grande femme de soie noire

Dans les regards brillent encore pour $\underline{\text { moi }}$

Et tous ses yeux peut-être éteints depuis longtemps. ${ }^{163}$

Cette multiplication donnée à la symbolique du feu peut refléter la prépondérance et l'importance de cet élément fondamental. Ici, le feu a la puissance de cristalliser la femme et la rendre beaucoup plus lumineuse et luisante. En effet, cet acte d'alchimie nous rappelle l'image baudelairienne du poète-alchimiste. Enfin, le feu est capable non pas uniquement de transformer la femme en un élément minéral, selon l'imagination matérielle de Jaccottet, mais aussi de rendre sa poésie plus passionnée et plus vivante. "Toute éloquence passionnée est une éloquence enflammée. Il faut toujours un peu de feu pour que les métaphores des autres éléments soient vives et clairs. La poésie multicolore est une flamme qui se colore des métaux de la terre. ${ }^{164}$

\section{Conclusion:}

Philippe Jaccottet, bien qu'il soit un poète-paysagiste; or nous pouvons dire que c'est une figure d'écrivain en rupture avec le romantisme. Sa relation avec la Nature n'est pas de la façon des poètes romantiques. Elle est beaucoup plus compliquée. C'est plutôt une méditation réflexive, à la fois philosophique et poétique, sur le monde sensible et ses éléments. A la fin de cette étude, il y a trois remarques à citer: d'abord, Jaccottet a réussi à ressusciter une thématique de la philosophie ancienne (les quatre éléments fondamentaux), et la transformer en une imagerie moderne; ensuite, il a 
donné la possibilité de réconcilier le monde imaginaire voire sensible et le monde matériel et minéral, et les exemples sont fréquents: femme-feu, airlumière, terre-mère et eau-mort. En fin de compte, notre poète a pu faire de la mythologie ancienne grecque ou persane (Prométhée, Le Phénix et Empédocle) des mythes modernes; qui conviennent à son aventure de la création poétique contemporaine.

Quatre éléments sont maintenant en jeu; mais Jaccottet marque sa préférence pour l'air: comme nous avons vu que tout est suspendu dans l'air: la montagne, la maison, la femme, et même les autres éléments fondamentaux. En conséquence, chez notre poète aérien, la démarche poétique semble se diriger vers l'intervalle, l'incertitude et surtout l'insaisissable. Il était évident d'emblée que l'incertitude constitue le sommet de la création poétique de Jaccottet. Nous venons de remarquer que tout au long de ses œuvres, il s'agit d'une poétique du presque, du comme si et d'approximation en approximation: c'est plutôt l'aventure d'être vivant ou la poésie de l'incertitude. Mais, cette incertitude et cette contradiction, qui se trouvent dans l'expression de "l'imagination matérielle", ne sont pas en fait gratuites: chez lui, c'est une manière d'exprimer le fait d'être au monde, dire le monde ou bien de le réorganiser; c'est la tâche grandiose de la poésie à travers les vers selon Jaccottet. L'aventure de la création poétique ne constitue pas pour lui une fin en soi. Elle a deux étapes nécessaires : d'abord, il s'agit d'une poésie du dépassement et de la traversée du négatif du monde. Ensuite, le poème s'impose comme une nécessité pour exprimer un état d'âme et un état d'esprit. Enfin, nous pouvons fixer, dans l'imagination matérielle de Philippe Jaccottet, une loi des quatre éléments: l'air, la terre, l'eau et le feu; qui serons désormais une phénoménologie propre à lui.

\section{Bibliographie:}

-Euvres de Philippe Jaccottet.

-Philippe Jaccottet: Poésie 1946-1967, Préface de Jean Starobinski, Gallimard, Paris, 1971.

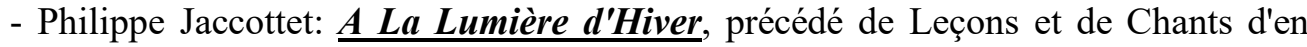
bas, Gallimard, Paris, 1977.

- Philippe Jaccottet: Pensées sous les nuages, Gallimard, Paris, 1983.

-Ouvrages critiques de Gaston Bachelard:

- Gaston Bachelard : L'Air et les songes- Essai sur l'imagination du mouvement, José Corti, Paris, 1983.

- G. Bachelard : La Psychanalyse du feu, Folio - Gallimard, Pais, 1985.

-Gaston Bachelard: La Poétique de la rêverie, PUF- paris, 1986.

- Gaston Bachelard: Fragments d'une Poétique du Feu, PUF, Paris, 1988.

- Gaston Bachelard: L L'Eau et les Rêves - Essai sur l'imagination de la matière, José Corti, Paris, 2003.

-Ouvrages critiques généraux:

- Jean-Pierre Richard: Onze études sur la poésie moderne, Seuil, Paris, 1964. 
- Marie-Claire Dumas et autres :La poésie de Philippe Jaccottet. Collection Unichamp. Champion, Suisse, 1985.

- Michèle Monte: Nymphes,Barques et autres "lieux" dans l'aeuvre de Philippe Jaccottet, http://babel.revue. Org/2719, 1997.

- Jacqueline Michel: Jouissance des déserts dans la poésie contemporaine, Lettres modernes Minard, Paris-Caen., 1998.

\footnotetext{
${ }^{11}$ Philippe Jaccottet: 1971, "Poésie" 1946-1967, préface de Jean Starobinski, Gallimard, Paris, p. 99

${ }^{2}$ Philippe Jaccottet: 1971, p. 103

${ }^{3}$ Philippe Jaccottet: 1971, p. 72

${ }^{4}$ Philippe Jaccottet: 1977, A la lumière d'hiver, "Leçons", Gallimard, Paris, p. 11

${ }^{5}$ Philippe Jaccottet: 1977, P.86

${ }^{6}$ Jean-Pierre Richard: 1964, Onze études sur la poésie moderne, Seuil, Paris, p.326

${ }^{7}$ Philippe Jaccottet: 1971, p. 85

${ }^{8}$ Gaston Bachelard: 1983, L'air et les songes- Essai sur l'imagination du mouvement, José Corti, Paris,p.55

${ }^{9}$ Philippe Jaccottet: 1971, p. 72

${ }^{10}$ Philippe Jaccottet: 1971 , p. 75

${ }^{11}$ Philippe Jaccottet: 1971, p. 99

12 Philippe Jaccottet: 1971, p. 63

${ }^{13}$ Philippe Jaccottet: 1971, p. 111

${ }^{14}$ Philippe Jaccottet: 1983, Pesées sous les nuages, Plaintes sur un compagnon mort, Gallimard, Paris, P.57

${ }^{15}$ Jacqueline Michel: 1998, Jouissance des déserts dans la poésie contemporaine, Lettres modernes Minard, Paris-Caen. P.84

${ }^{16}$ Gaston Bachelard: 1983, p.18, 23,111

${ }^{17}$ Philippe Jaccottet: 1977, Chants d'en bas, p.49

${ }_{18}$ Marie-Claire Dumas et autres:1985, La poésie de Philippe Jaccottet. Collection

Unichamp. Jean-Marc Sourdillon " Nuages de novembre ou le langage de la fidélité",

Champion, Suisse, p.209

${ }^{19}$ Philippe Jaccottet: 1971, La promenade à la fin de l'été, p. 85

${ }^{20}$ Philippe Jaccottet: 1971, Fin d'hiver, p.95

${ }^{21}$ Philippe Jaccottet: 1977, A la lumière d'hiver, p.86

22 Philippe Jaccottet: 1977, Leçons, p.15

${ }^{23}$ Philippe Jaccottet: 1977, p.29

${ }^{24}$ Philippe Jaccottet: 1971, Le Livre des morts, p.91-92

${ }^{25}$ Philippe Jaccottet: 1977, P.90

${ }^{26}$ Philippe Jaccottet: 1977, P.71

${ }^{27}$ Philippe Jaccottet: 1971, P.131

${ }^{28}$ Philippe Jaccottet: 1971, P.132

${ }^{29}$ Philippe Jaccottet: 1971, La Semaison, P.40

${ }^{30}$ Philippe Jaccottet: 1977, Autres chants, p.71

${ }^{31}$ Philippe Jaccottet: 1983, Plaintes sur un compagnon mort, p.69

${ }^{32}$ Gaston Bachelard:2003, L'Eau et les Rêves - Essai sur l'imagination de la matière, José Corti-Paris, p.35

${ }^{33}$ Gaston Bachelard:1986, la poétique de la rêverie, $\mathrm{PUF}$, paris, p. 146

${ }^{34}$ Philippe Jaccottet: 1983, Le Mot joie, p.38

${ }^{35}$ Gaston Bachelard:2003, p.45
} 
${ }^{36}$ Philippe Jaccottet: 1977, A la lumière d'hiver, p.78

${ }^{37}$ Gaston Bachelard:2003, p. 150

${ }^{38}$ Philippe Jaccottet: 1971, Ninfa, p.36

${ }^{39}$ Michèle Monte:1997, Nymphes, Barques et autres "lieux" dans l'œuvre de Philippe

Jaccottet, http://babel.revue. Org/2719, p.6

${ }^{40}$ Philippe Jaccottet: 1971, Notes pour le petit jour, p.53

${ }^{41}$ Philippe Jaccottet: 1971, Airs, p.111

${ }^{42}$ Philippe Jaccottet: 1977, p.29

${ }^{43}$ Gaston Bachelard:2003,p.p80/96

${ }^{44}$ Philippe Jaccottet : 1983, A Henry Purcell, p.70

${ }^{45}$ Philippe Jaccottet: 1971, La Seine le 14 mars 1947, p.42/43

${ }^{46}$ Gaston Bachelard:2003, p.108

${ }^{47}$ Philippe Jaccottet: 1971, Portovénère, p.31

${ }^{48}$ Gaston Bachelard:2003, p. 175

${ }^{49}$ Gaston Bachelard: 1983, p.227

${ }^{50}$ G. Bachelard : 1985, La psychanalyse du feu, Folio - Gallimard, Pais, p.174

${ }^{51}$ Philippe Jaccottet: 1971, Leçons, p.180/181

${ }^{52}$ Gaston Bachelard: 1988, Fragments d'une Poétique du Feu, PUF-Paris, P.63/69

53 Philippe Jaccottet: 1983, Pensées sous les nuages, p.21

${ }^{54}$ Philippe Jaccottet: 1971, Au petit jour, p.57

${ }_{55}^{55}$ Philippe Jaccottet: 1971, Au dernier quart de la nuit, p.104/105

${ }^{56}$ G. Bachelard :1985,p.35

${ }^{57}$ Gaston Bachelard: 1988, p.137/149

${ }^{58}$ Philippe Jaccottet: 1977, Leçons, p.11

${ }^{59}$ Philippe Jaccottet: 1971, p. 180

${ }^{60}$ Philippe Jaccottet: 1971, p.92

${ }^{61}$ Gaston Bachelard: 1988, p.107

${ }^{62}$ Philippe Jaccottet: 1977, Chants d'en bas, p. 43

${ }^{63}$ Philippe Jaccottet: 1977, A la lumière d'hiver, p. 85

${ }^{64}$ G. Bachelard : 1983, p. 153 